\title{
Câmera e câmara: un caso di variazione lessicale in portoghese
}

\section{Câmera and câmara: a case of lexical variation in Portuguese}

\author{
Ricardo Cavaliere \\ Universidade Federal Fluminense (UFF), Niterói, Rio de Janeiro, Brasil. \\ cavaliere@oi.com.br
}

\begin{abstract}
Questo articolo tratta dell'origine etimologica della doppia câmara e câmera nel lessico del portoghese, con uno studio delle loro varianti semantiche e del loro uso nel testo scritto in prospettiva diacronica. I paronimi in questione sono casi di allotropia, arrivati dall'importazione del lessico delle lingue moderne, oltre a dalle forme ereditarie della tradizione latina. L'articolo riporta, inoltre, un breve studio comparativo con l'obiettivo di analizzare gli aspetti semantici che i termini godono in altre lingue romanze.
\end{abstract}

Parole chiave: lessico; portoghese; allotropi.

Abstract: This paper studies the etymological origin of the pair câmara and câmera in the lexicon of Portuguese language, with a study of their semantic variants and their use in written texts in diachronic perspective. The paronyms at issue are examples of doublets that have arrived from the vocabulary of modern languages, in addition to hereditary forms of the Latin tradition. The article also offers a short comparative study with the objective of analyzing the semantic aspects that these particular words have in other romance languages.

Keywords: lexicon; Portuguese; doublets.

Recebido em 27 de julho de 2014. Aprovado em 15 de outubro de 2014. 
Fatto comune nelle lingue moderne, la doppia forma lessicale delle parole di significato esterno attrae l'attenzione dei lessicologi impegnati negli studi diacronici. Il fenomeno detto allotropia è abbastanza conosciuto nel piano degli studi diacronici delle lingue romanze, definito come due o più parole distinte che vengono dallo stesso etimo latino. $\mathrm{Ci}$ sono tre cause della coesistenza di forme allotropiche comunemente registrate nella storia delle lingue romanze: la via popolare, la via erudita e l'importazione straniera. Così, una data parola latina che è entrata nel lessico della lingua destinataria attraverso la via popolare, e quindi è stata modificata fonetica e morfologicamente, in ottemperanza al cambio linguistico nel corso del tempo può essere inserita un'altra volta in una sincronia posteriore attraverso la via erudita, oppure attraverso l'importazione straniera, fatto da cui deriva la coesistenza di forme allotropiche nelle sincronie future.

In portoghese si possono osservare innumerevoli casi di allotropia a causa del rientro lessicale per mezzo delle vie riferite, secondo occorre in frigidum $>$ (pop. $)^{1}$ frio; frigidum $>$ (erud.) frígido; hospitalem $>$ (pop.) esprital; hospitalem > (erud.) hospital; hospitalem $>$ (fr.) hôtel $>$ hotel etc. Recentemente, è entrata nel vocabolario portoghese la parola tablet $<$ (ingl. tablet), con pronuncia parossitona, un doublet della forma popolare tábua $<$ tabulam, della forma erudita tábula $<$ tabulam e della forma importata tablete $<$ (fr.) tablette $<$ tabulam.

Va notato che l'applicazione del termine allotropo può essere contestata in relazione a câmara e câmera, poiché queste forme sono già registrate nel lessico del latino. Loro sono, infatti, la variazione fonetica e morfologica risultante dall'importazione etrusca, siccome sarà specificato di seguito. Tuttavia, per quanto riguarda il portoghese, l'ipotesi di allotropo è consolidata, dal momento che le parole entrano nel lessico in tempi diversi e per vie diverse. In altre parole, non abbiamo câmara e câmera alla luce della variazione lessicale del latino, ma a causa delle diverse vie di ingresso per quanto riguarda questi elementi lessicali.

Si sa che le varianti contemporanee di un vocabolo portoghese si devono ordinariamente a fatti ristretti al piano fonetico, come accade nei casi in cui coesistono forme con $b$ o $v$-assobio, assovio - decorrenti dalla mutazione della consonante occlusiva, e nelle coppie di alternanza vocalica in sillaba atona - dezasseis, dezesseis -, in cui hanno agito forze assimilatorie nel corso prosodico della parola. Tuttavia ci sono situazioni in cui la coesistenza di forme analoghe si deve alla doppia 
entrata di certi termini nel lessico del portoghese, spesso da fonti distinte e in epoche diverse. È questo il caso di corredor - luogo di passaggio - e corredouro, di cui il primo proviene dall'italiano antico corridore (CUNHA, 1994), già registrato nella seconda edizione del dizionario di Antônio de Morais Silva (1813), e il secondo si trova registrato nel secolo XII (cfr. VITERBO, 1965) come derivato da cürrěre. Nelle coppie di parole divergenti in cui figurano una forma erudita e un'altra ereditaria, di solito si trova grande distinzione semantica nella lingua contemporanea, come in artelho e artigo, plano e chão, ecc. Nel caso di câmara e câmera, la coppia alla quale ci dedicheremo in questo breve studio, la schematizzazione delle varianti sembra non si possa spiegare facilmente, dato che l'uso delle due forme oggi si configura indistinto in alcuni casi e, in altri, compulsoriamente distinto, dovuto alla loro polisemia.

Ad esempio, si sente dire che ultimamente i deputati poco si sono fatti presenti (come di solito succede) alle riunioni della Câmara, fatto registrato dalle câmeras dei fotogiornalisti. La convivenza dei due termini paronimici nei testi contemporanei dà spazio a una supposta distinzione di significati, come se costituissero due vocaboli differenti, il primo indicante "stanza" o "spazio" ristretto, dovuto alla sua peculiarità, all'accesso delle persone - câmara nupcial, câmara mortuária, câmara-ardente, ecc. - senso che, metonimicamente, si allarga ai consigli e organi collegiali rappresentativi - câmara comercial, câmara de deputados, câmara cível, ecc.

Apparentemente, l'attuale significato di câmara implica necessariamente una nozione di spazio fisicamente delimitato, a cui si aggiunge l'idea di reclusione o privacità di uso. In questo caso, il termine viene usato nel linguaggio medico per designare le varie cavità e spazi del corpo, così come la cavità o spazio intercellulare (cfr. HOUAISS, 2001; FERREIRA, 2000). L'espressione câmara de sangue, che sta perdendo presenza nel linguaggio medico contemporaneo, ma ancora viene registrata nei lessici del portoghese, dello spagnolo e dell'italiano, conduce all'idea di "diarrea sanguinolenta", sicuramente risultante da ragionamento metaforico. Seguendo questa linea, Moraes Silva offre il registro di "evacuazione del ventre" o "escremento humano" (SILVA, 1813), il che rafforza il fatto che abbia avuto un valore semantico espressivo perlomeno fino alla fine del Settecento in portoghese. In fiorentino, come in altri dialetti italici, si registra l'espressione andare a chamera, come in questo brano di Zucchero Bencivenni: "l'acieto à questa natura, che s'elli truova lo stomaco pieno sì fa bene andare 
a chamera, e s'elli il truova voto sì ristringnie" (CONSIGLIO, 2006). Si osservi, a questo proposito, il digramma ellenico, che denuncia nella grafia l'origine della parola. A questo riguardo, la dialettologia diacronica italiana offre una proficua variazione ortografica: camara, camera, cammera, cammara, chamera, kammora, ecc., come ci informa il prezioso e utilissimo Tesoro della lingua italiana delle origini, che oggi può essere consultato da qualsiasi luogo del pianeta grazie a Internet.

Si noti, in special modo, che la metonimia in casi come câmara de comércio e câmara de vereadores - che sposta l'attribuzione del contenente verso il contenuto, o più esattamente verso il gruppo di persone che per obblighi di mestiere si riuniscono alla câmara - può assumere matrici distinte quando viene a significare il tutto per la parte. È questo il caso di câmara (câmera) fotográfica e câmara (câmera) cinematográfica, concetto relativo a qualsiasi dispositivo costituito da una specie di scatola o scompartimento chiuso, o quasi chiuso, con un'apertura attraverso la quale raggi luminosi vengono captati per registrare un'immagine su di una pellicola chimica.

A sua volta, câmera non gode di uno spettro semantico così ampio, dato il suo uso contemporaneo si limita all'indicazione del citato dispositivo fotografico o cinematografico, con naturale estensione verso l'individuo che manovra professionalmente questo dispositivo, caso in cui vince sul termine câmara per frequenza d'uso. Sicuramente alcune espressioni isolate come música de câmera concorrono con la corrispondente scritta con la lettera $a$, le quali si spiegano con il surriferito rientro di certi termini nel lessico del portoghese mediante prestiti delle lingue moderne. Come con accortezza afferma Antenor Nascentes (NASCENTES, 1966), in música de câmera dobbiamo riconoscere l'influsso dell'italiano camera, che nella lingua di Dante presenta uno speciale rilievo semantico perché indica la sala del consiglio per scopi musicali. La presenza dell'italiano, tra l'altro, è flagrante nel vocabolario della musica classica in tutte le lingue occidentali. Da questo termine abbiamo anche ereditato il termine camerista, italianismo che indica il musicista specializzato in música de câmera, e camerata, piccolo gruppo strumentale. Per via di regola, però, oggi non troviamo nel portoghese brasiliano, perlomeno nella lingua standard, la forma con la lettera $e$ nel senso di "stanza" o "spazio", ad eccezione delle espressioni isolate già commentate in questo testo.

Una curiosità iniziale riguarda questa notevole preferenza per câmera come descrizione del dispositivo fotografico. Invero, il fatto rivela 
che, nonostante coesistano nel lessico del portoghese, i due termini che ora studiamo mantengono una relazione di disputa per la preferenza dei parlanti in ognuna delle accezioni possibili, in tal modo che la naturale ripetizione e solidificazione dell'uso di una di esse finisce col sopprimere l'impiego dell'altra. Le conseguenze della concorrenza lessicale sono abbondantemente esemplificate in portoghese, come nelle coppie mais-chus, depressa-asinha e tante altre, in cui il parlante semplicemente ha scelto una delle forme semanticamente equivalenti, portando ad una progressiva elisione dell'altra.

Nel caso della progressiva preferenza di câmera per câmara, in riferimento all'accezione della macchina fotografica, almeno nel portoghese del Brasile, metto in risalto l'ipotesi che ci sia un influsso dell'inglese, come vedremo avanti, che registra soltanto il nome primitivo con la $e$, e specificamente in questa accezione. Il fatto sicuramente si deve all'intenso movimento mercantile dei tempi moderni accanto al fatto che gli strumenti sopraccitati siano fabbricati in paesi stranieri, che preferiscono denominarlo nella forma inglese, visto che innegabilmente l'inglese è la lingua franca dell'industria e del commercio. Con ciò, si è popolarizzato il vincolo di câmera al senso specifico del dispositivo fotografico o cinematografico.

Dando voce agli etimologi del latino, è ormai un dato di fatto l'origine di camĕra, ae nel greco $\kappa \alpha \mu \alpha \rho \alpha$. Émile Boisacq ci insegna che il termine esprime di solito nel greco i significati di "volta di una stanza", "volta arcata", "soffitto del letto" e "tetto di carrozza" (BOISACQ, 1923). In tutti i casi, predomina l'idea di forma arcata. Nel latino letterario, tra l'altro, regna pacifica questa forma con la $\breve{e}$, il cui senso inizialmente sarebbe stato limitato alla riferita nozione di volta. Secondo Corominas, l'accezione romanza di "stanza" o "alcova" che, come abbiamo visto, ha già antecedenti nel greco, si trova in Sant'Agostino e Casiodoro (COROMINAS, 1954).

Il termine, sicuramente, è entrato nella lingua di Cicerone per influssi degli ellenismi incorporati al lessico latino com intermediazione etrusca. Secondo Ernesto Farias (FARIAS, 1970), questi influssi dell'etrusco costituiscono l'unica spiegazione plausibile per le varianti di grafia delle parole greche in latino, che a volte si trascrivono con la $p$ parole originalmente scritte con la $\beta$, a volte con la $b$ termini in origine scritti con la $\pi$, tra i vari cambiamenti. Questo perché l'etrusco non aveva le sonore $/ \mathrm{b} /, / \mathrm{d} / \mathrm{e} / \mathrm{g} /$, fatto che imponeva ai termini ellenici con tali consonanti grandi variazioni di grafia quando entravano nel latino: gr. 
kubernãn, lat. gubernare; gr. pyrrós, lat. burrus; gr. amorga, lat. amurca (FARIAS, 1970, p. 22).

Solo dopo il termine dell'occupazione etrusca tra i secoli V e IV a.C., i romani poterono mantenere diretto contatto con le città greche già fondate nella Penisola Italica. Da questo momento, l'influenza ellenica nel latino si intensificò in modo esponenziale, facilitando l'entrata di prestiti che man mano si adattavano con una grande facilità al sistema fonetico latino. La mutazione fonetica dal gr. $\kappa \alpha \mu \alpha \rho \alpha$ al latino camera sembra abbia seguito una regola generale di dissimilazione che attinge la vocale della sillaba atona interna. Così, come in kamára $>$ camera, si registrano altri casi analoghi di dissimilazione: gr. Taras, Tárantos, lat. Tarentum; gr. kóthornos, lat coturnus (cfr. FARIAS, 1970:24).

Comunque, è molto probabile l'ipotesi del fatto che l'entrata di $\kappa \alpha \mu \alpha \rho \alpha$ si sia avuta con la conservazione della vocale bassa interna, anche se per un breve periodo. Affinché si ammetta la regola di mutazione surriferita, inevitabilmente bisogna accettare una forma anteriore ancora con la $a$ in latino, di modo che si possa normalmente inquadrare il cambiamento di $\breve{a}$ in $\breve{e}$ all'interno della regolarità che attingeva la vocale bassa in sillabe libere. La soluzione offre una lieve avversità se osserviamo che, in sillaba anteriore libera, la $\breve{a}$ passa normalmente a $\breve{e}$ e dopo a $\breve{l}$, come in *perfacio $>$ *perfercio $>$ perficio e abago $>$ abego $>$ abigo (cfr. FARIAS, 1970, p. 182). Nel caso di caměra, quindi, la $\breve{a}$ precedente alla $r$, proveniente o no da rotacismo, passa a $\breve{e}$, come successe in *transdade $>*$ tradare $>$ tradere. È di dovere notare ancora che, per convalidare questa ipotesi di mutazione fonetica per dissimilazione, dovremo allo stesso modo e obbligatoriamente ammettere un previo spostamento sistolico che trasformò il termine ellenico in un proparossitono latino.

Un'altra questione attinente alla presenza di caměra e camăra in latino concerne l'impiego delle parole nelle variabili d'uso, tanto nella lingua orale quanto in quella scritta. Si osservi che la forma camăra viene attribuita senza dubbio al latino volgare, senza che però venga spiegato chiaramente il motivo per cui questa variante con la $\breve{a}$ si sia rafforzata negli usi popolari, in dissonanza con la forma con la $\breve{e}$, che si è consolidata nella lingua scritta. In principio, il fatto rafforza la tesi secondo la quale, nonostante per breve periodo, la vocale bassa interna dell'etimo greco si mantenne in latino prima di dissimilarsi in $\breve{e}$. Da questo avrebbero seguito il loro corso parallelo la primitiva forma camăra e l'alterata forma caměra, di cui quest'ultima veniva usata in registri letterari, conferendogli maggior prestigio. 
L'Appendix Probi, come sappiamo, registra la correzione caměra non cammăra, netta evidenza di maggior prestigio della forma con la ĕ nel sermo litterarius. Vengono registrate, tuttavia, testimonianze di ambedue le forme in qualche testo scritto, come ci informa l'erudito lessicografo Wilhelm Freund (FREUND,1860, p. 399), fatto questo che ci sembra provare l'esistenza di una forma primitiva nel lessico latino con la vocale bassa interna. Seguendo la stessa linea, si rivela illustrativa la lezione di Juan Corominas, secondo il quale la forma caměra è quella normalmente usata in latino scritto, ma camăra compare non solo come volgarismo, ma anche in testi di autori ispanici come Seneca (cfr. COROMINAS, 1954).

È importante mettere in risalto, tra l'altro, che i registri di questa forma non sono così rari in testi di tematica popolare, come l'Itinerario terrae sanctae, di Admnanus: "Ecclesiae interior domus sine tecto, et sine camara, ad coelum sub aere nudo patet" (CANGE, 1937:38). Inoltre bisogna aggiungere che durante il lungo periodo anteriore al secolo I a.C., quando fiorisce in magnitudine la letteratura latina, non si poteva parlare di differenze lessicali espressive tra il sermo urbanus e il sermo litterarius. In realtà, non bisogna dimenticare che la fonte del vocabolario di prestigio nella norma colta scritta sempre era sempre stata il vocabolario popolare sedimentato dall'uso esemplare durante i secoli (MAURER JR, 1962).

La coesistenza di varianti diastratiche del latino orale in culti religiosi e nelle commedie teatrali, soprattutto, favoriva il sorgere di forme lessicali in concorrenza, fatto che può spiegare il graduale cambiamento di camăra in caměra senza che il termine originale si sia totalmente eliso.

Nella sua edizione dell' Appendix, Serafim da Silva Neto (SILVA NETO, 1946, p. 231) si occupa singolarmente della nasale geminata nella forma volgare - a suo giudizio, una pronuncia espressiva, simile ad altri casi come in *brutto $>$ bruto; ${ }^{*}$ burriccu $>$ burrico; camello $>$ camelo, ecc. senza però tracciare perlomeno una riga sulla questione della vocale atona interna. Comunque, la presenza della correzione camĕra non cammăra nell'Appendix fa supporre che i grammatici latini credessero fosse la forma con la $\breve{a}$ una mutazione secondaria della forma con la $\breve{e}$, creata dal parlante come risultato di un processo di assimilazione vocalica. Questa ipotesi, così, gareggia con quella anteriormente riferita - del fatto che camăra abbia preceduto caměra - come spiegazione ammissibile della presenza delle due varianti nel lessico del latino. Inoltre, non si elimini totalmente l'improbabile pronuncia camăra per ipercorrezione, o 
possibilmente per eruditismo in un tentativo di recuperare l'etimo greco: "quod est graecos imitari" (FORCELLINI, 1940).

Sarà giustamente questa presenza duale che giustificherà la disseminazione eterologa di parole ereditarie nelle diverse lingue moderne che hanno ricevuto l'etimo latino, qui con il radicale in $\breve{e}$, là con il radicale in $\breve{a}$. Nel primo caso, si registra il flusso verso il nord, in cui la $\check{e}$ prevale nel francese chambre, nel catalano cambra, nel tedesco Kammer, nell'inglese camera, in quest'ultimo soltanto con il senso di macchina fotografica o cinematografica. Riguardo specificamente al francese, è evidente che la forma chambre deriva dal latino caměra (cfr. LEBRUN; TOISOUL, 1937), fatto questo che rafforza la tesi secondo la quale ambedue le forme camĕra e camăra coesistevano nelle vertenti diatopiche del latino volgare, dato che chambre è un termine ereditario. Si metta in risalto il fatto che, tra l'altro, non si può scartare lo spostamento di ambedue le forme verso il francese antico, a giudicare dai derivati caméral e camériste in confronto a camarade (LITTRÉ, 1956).

Per ciò che concerne le vertenti del latino che si espansero verso ovest, specificamente quelle che si trovano nelle fonti dei dialetti meridionali dell'italiano, e quella che si è spostata verso la Penisola Iberica dando origine allo spagnolo e al portoghese nonostante ci siano registri di ambedue le forme, quella considerata volgare (sp. cámara, port. câmara) sembra costituire la fonte delle parole ereditarie. Nell'italiano moderno sicuramente predomina camera, ma nei dialetti della Corsica e della Sicilia il registro ordinario è di camara (cfr. COROMINAS, 1954). In questo modo, la variante con la $e$ in queste lingue può inizialmente essersi iscritta nello spazio che generalmente occupano nel linguaggio colto gli eruditismi, con una posteriore espansione nella lingua corrente. In spagnolo, ad esempio, la maggioranza dei termini che compongono la famiglia etimologica deriva dal radicale in $\breve{a}$ : camarada (colui che dorme nella stessa cámara), camaranchón (luogo della casa dove si tengono cose vecchie), camarera (cameriera) e camarero (capo della camera del re), camarin, tra le altre. La presenza del derivato camerino, secondo Corominas, si deve all'influenza dell'italiano (COROMINAS, 1954).

Quindi non è senza motivo che il Diccionario de la lengua española, della Real Academia (REA, 1984) metta in risalto la forma cámara come quella principale, attribuendogli tutti i sensi conosciuti e vincolandola all'etimo latino camăra. Dal punto di vista semantico, tuttavia, si rivela sorprendente che nell' eccellente dizionario il principale 
significato sia quello di "sala o stanza principale di una casa", malgrado la sua conosciuta origine come "stanza di riposo o di reclusione". Comunque, si verifica che la primitiva nozione di "stanza in forma arcata o a volta" si è persa nel tempo.

Infine, per ciò che riguarda la presenza della coppia in portoghese, si deve concludere che la lotta per la preferenza del parlante viene da lunga data, e da cui è risultata una riserva di significanza per ogni parola nel decorrere del tempo. Bisogna comunque registrare ambedue le parole in testi portoghesi fin dal secolo XIV, con sensibile prevalenza della forma con la a (MACHADO, 1952; SILVA, 1813), che fin dagli inizi ebbe il priviliegio di designare la "stanza per dormire". Curiosamente, si riferiva all'espressione câmara cerrada, che significava la "somma incerta che il marito promette alla moglie di tappezzeria [di Arras], o forse tutto il necessario per l'arredamento della camera della moglie, senso in accordo alla legge del 9 febbraio 1643" (SILVA, 1813). Questo percorso semantico è un'eredità della liturgia matrimoniale romana, a giudicare dal registro di camăra come "dotalitium uxoris" nel Glossario di du Cange (CANGE, 1937).

Il vocabolario di Bluteau ratifica la presenza delle due forme in portoghese, stabilizzata per lungo periodo. Parlando di questo, Bluteau già ponderava una distinzione semantica ben delineata degli usi di cui ambedue godono in portoghese, conferendo a câmara il senso particolare di "la casa in cui si dorme". Invece, nel senso di ente amministrativo o ripartizione pubblica, il chierico londinese indica l'uso indistinto di câmara o câmera: "As casas, \& o Tribunal, em que o Presidente, Vereadores, \&c. se ajuntão para tratar dos negócios concernentes ao bem publico de hua cidade [...]". Da Jacinto Freire estrae Bluteau il seguente passo: "Pedio vinte mil, Pardaos à Camera de Goa (BLUTEAU, 1712, p. 69).

L'evoluzione semantica dei termini in portoghese ci rivela una progressiva predilezione per la forma con la $a$, che si trova oggi in tutti i sensi in un modo generale. Il dizionario di Aurélio Buarque de Holanda (FERREIRA, 1970) conferisce 16 accezioni per câmara, che vanno dal senso di "vano o stanza di una casa e, in special modo, la stanza da letto" fino a "persona che opera la macchina da presa del cinema o televisione", il che rivela la grande area semantica di uso della forma proveniente dal latino volgare. Si dica lo stesso del prezioso lessico di Antônio Houaiss, nonostante questo sia meno dettagliato in espressioni o lessie in cui consta la nostra parolina. 
Con tutto ciò, ambedue i lessici brasiliani rivelano una mancanza per ciò che concerne la descrizione di usi quando attestano la forma câmera nel portoghese contemporaneo, come semplice variante di câmara, senza che vengano distinte le rigorose restrizioni che la forma con la $e$ oggi subisce nell'area semantica della terminologia amministrativa o giuridica. Infatti, nel portoghese del Brasile, oggigiorno non sono ammesse espressioni come "Câmera dos Deputados" o "Câmera Cível, Câmera Criminal" - nel senso di "organo dei tribunali" - giacché l'uso normativo impone la forma con la $a$ come esclusiva. Il dizionario di Houaiss, in verità, avverte che câmera "è di uso corrente specialmente nelle accezioni di 'dispositivo o apparecchio ottico' in cinema, foto e tv" (HOUAISS, 2001), il che effettivamente accade.

A questo proposito, il recente Dicionário de usos di Francisco da Silva Borba (BORBA, 2002), che dovrebbe coprire un ruolo più accurato in questa specifica area di usi linguistici, lascia a desiderare quanto l'esatta descrizione semantica delle due forme lessicali.

Infine, la surriferita preferenza di câmera nell'accezione di macchina fotografica, macchina da presa ecc., a cui ci siamo già riferiti come probabile rientro per prestito dall'inglese, costituisce un esempio preciso di questo processo di flussi e riflussi che l'intenso scambio lessicale sta imponendo alle lingue contemporanee. L'ipotesi si rafforza se osserviamo che lo stesso fatto è sintomatico nel tedesco, in cui, come per il vocabolo Kammer, oggi si registra la forma Kamera, prestata dall'inglese camera, per designare specificamente la macchina fotografica o da presa (HOEPNER, 2001, p. 893).

Crediamo che ulteriori studi circa altri tipi di doppioni possano contribuire ad una migliore comprensione della presenza di câmara e câmera non solo in portoghese, ma anche in altre lingue romanze. D'altra parte, la valutazione semantica di queste coppie può rivelare i criteri che il parlante prende in considerazione per assegnare un particolare significato ad uno dei componenti della coppia lessicale. Va notato, inoltre, che le forze relative alle relazioni sociali contemporanee, che danno la possibilità agli scambi lessicali con maggiore frequenza e velocità, soprattutto nel settore del commercio internazionale, possano più convincentemente spiegare la coesistenza di forme allotropiche con uguale valore semantico nel lessico delle lingue moderne. 


\section{Riferimenti}

BLUTEAU, R. Vocabulário portuguez e latino. Coimbra: Collegio das Artes da Companhia de Jesu, 1712.

BOISACQ, É. Dictionnaire étymologique de la langue grecque. 2 ed. Heidelberg, Paris: Carl Winter's, Librairie C. Klincksieck, 1923.

BORBA, F. da S. Dicionário de usos do português do Brasil. São Paulo: Ática, 2002.

CANGE, D. du. Glossarium mediae et infimae latinatis. Paris: Librairie de Sciences et des RTS, 1937, T. II.

CONSIGLIO NAZIONALE DELLE RICERCHE. Tesoro della lingua italiana delle origini. Disponível em: $<$ http://tlio.ovi.cnr.it $>$. Acesso em: 24 mai. 2006.

COROMINAS, J. Diccionario crítico etimológico de la lengua castellana. Madrid: Gredos, 1954, v. 1.

CUNHA, A. G. da. Dicionário etimológico nova fronteira da língua portuguesa. 2 ed. Rio de Janeiro: Nova Fronteira, 1994.

DE MAURO, T. Grande dizionario italiano dell'uso. Torino: UTET, 1999. FARIA, E. Fonética histórica do latim. 2 ed. Rio de Janeiro: Acadêmica, 1970. FERREIRA, A. B. de H. Novo dicionário da língua portuguesa. Rio de Janeiro: Nova Fronteira, 1970.

FORCELLINI, A. Lexicon totius latinarum. Padua: T. Seminarii, 1940. 4v. FREUND, G. Grand dictionnaire de la langue latine. Traduit en français par N. Theil. Paris: Librairie de Firmin Didot Frères, Fils et Cie., 1860.

HOUAISS, A. Dicionário eletrônico Houaiss da língua portuguesa. Rio de Janeiro: Instituto Antônio Houaiss, Editora Objetiva, 2001. Versão 1.0. LEBRUN, L.; TOISOUL, J. Dictionnaire étymologique de la langue française. 10 ed. Paris: Librairie Fernand Nathan, 1937.

LITTRÉ, É. Dictionnaire de la langue française. Paris: Jean-Jacques Pauvert Editeur, 1956.

MACHADO, J. P. Dicionário etimológico da língua portuguesa. Lisboa: Editorial Confluência, 1952, t. 1.

MAURER JR., T. H. O problema do latim vulgar. Rio de Janeiro: Acadêmica, 1962. 
NASCENTES, A. Dicionário etimológico resumido. Rio de Janeiro: MEC/INL, 1966.

PANTOJA, M. J. Camara non cammara (app.pr.84): la geminada latina -mm- en eukera. In: GARCÍA-HERNÁNDEZ, Benjamín. Latín vulgar y tardio: homenaje a Veikko Väänänen (1905-1997). Madrid: Ediciones Clásicas, 2000. p. 157.

PIDAL, R. M. Léxico hispánico primitivo (siglos VIII al XII). Redactado por Rafael Lapesa e Constantino García. Madrid: Fundación Menendez Pidal/Real Academia Española, 2003.

REAL ACADEMIA ESPAÑOLA. Diccionario de la lengua española. 20 ed. Madrid: Real Academia Española, 1984, t. I.

SILVA NETO, S. da. Fontes do latim vulgar: o appendix probi. Rio de Janeiro: Imprensa Nacional, 1946

SILVA, A. de M. Diccionario da lingua portugueza recopilado dos vocabularios impressos até agora, e nesta segunda edição novamente emendado, e muito accrescentado. 2 ed. Lisboa: Typographia Lacerdina, 1813.

VITERBO, J. de S. R. de. Elucidario das palavras, termos e frases que em Portugal antigamente se usaram. Lisboa: Off. da Academia Real das Sciencias 1789. 